\title{
Phoneme Categorization and Discrimination in Younger and Older Adults: A Comparative Analysis of Perceptual, Lexical, and Attentional Factors
}

\author{
Sven L. Mattys \\ University of York
}

\author{
Odette Scharenborg \\ Max Plank Institute for Psycholinguistics, Nijmegen, The \\ Netherlands and Radboud University Nijmegen
}

\begin{abstract}
This study investigates the extent to which age-related language processing difficulties are due to a decline in sensory processes or to a deterioration of cognitive factors, specifically, attentional control. Two facets of attentional control were examined: inhibition of irrelevant information and divided attention. Younger and older adults were asked to categorize the initial phoneme of spoken syllables ("Was it $m$ or $n$ ?"), trying to ignore the lexical status of the syllables. The phonemes were manipulated to range in eight steps from $m$ to $n$. Participants also did a discrimination task on syllable pairs ("Were the initial sounds the same or different?"). Categorization and discrimination were performed under either divided attention (concurrent visual-search task) or focused attention (no visual task). The results showed that even when the younger and older adults were matched on their discrimination scores: (1) the older adults had more difficulty inhibiting lexical knowledge than did younger adults, (2) divided attention weakened lexical inhibition in both younger and older adults, and (3) divided attention impaired sound discrimination more in older than younger listeners. The results confirm the independent and combined contribution of sensory decline and deficit in attentional control to language processing difficulties associated with aging. The relative weight of these variables and their mechanisms of action are discussed in the context of theories of aging and language.
\end{abstract}

Keywords: speech perception, divided attention, dual-task, aging, lexical access

A central question in speech and hearing research concerns the extent to which the difficulties experienced by older adults in processing spoken language are due to an impoverishment of lower-level sensory processes. Sensory-decline approaches posit that age-related loss of audibility, coarser time resolution, and reduced spectral discrimination lead to deleterious cascaded effects on higher-order functions in both the short and the long term (e.g., Dubno, Dirks, \& Morgan, 1984; Humes, 1996; Schneider, Daneman, Murphy, \& Kwong-See, 2000; see also Gao, Levinthal, \& Stine-Morrow, 2012, in the visual modality). In this conceptualization, the unique contribution of cognitive deficits to declines in language processing is comparatively small (e.g., Murphy,

Sven L. Mattys, Department of Psychology, University of York, York, United Kingdom; Odette Scharenborg, Max Plank Institute for Psycholinguistics, Nijmegen, The Netherlands and Donders Institute for Brain, Cognition and Behaviour, Radboud University Nijmegen, The Netherlands.

This research was funded in part by the Max Planck International Research Network on Aging, Rostock, Germany (to Odette Scharenborg), by a visiting scholar grant from the Max Planck Institute for Psycholinguistics (to Sven L. Mattys), and by the Economic and Social Research Council (RES-062-23-2746 to Sven L. Mattys). We thank the student assistants of the Adaptive Listening Group of the Max Planck Institute for Psycholinguistics, Nijmegen, for testing our participants.

Correspondence concerning this article should be addressed Sven L. Mattys, Department of Psychology, University of York, Heslington, York, United Kingdom, E-mail: Sven.Mattys@york.ac.uk; or Odette Scharenborg, Centre for Language Studies, Radboud University Nijmegen, Erasmusplein 1, 6525 HT Nijmegen, NL. E-mail: O.Scharenborg@let.ru.nl
Daneman, \& Schneider, 2006; Schneider, Daneman, \& Murphy, 2005; Schneider, Pichora-Fuller, \& Daneman, 2010; see Guerreiro, Murphy, \& Van Gerven, 2010, for a review). This claim is important for both psycholinguistic theory and clinical intervention. From a theoretical viewpoint, the sensory-decline approach provides a basis for investigating the relationship between perception, cognition, and aging. From a clinical perspective, the sensorydecline hypothesis implies that restoration of acceptable language functions in older adults could be largely achievable through peripheral intervention (e.g., hearing aids and cochlear implants; see Pichora-Fuller \& Singh, 2006, for an appraisal of this possibility).

Although the sensory-decline approach has gathered a great deal of empirical support over the years, especially when adverse listening conditions such as noise, reverberation, and competing talkers are considered (e.g., Humes \& Christopherson, 1991; Humes, Nelson, \& Pisoni, 1991; Souza \& Turner, 1994; Takahashi \& Bacon, 1992), complex interactions between peripheral auditory, central auditory, and cognitive abilities have also been found (e.g., McCoy et al., 2005; Wingfield, Tun, \& McCoy, 2005). Specifically, some studies have shown enduring age effects on speech processing even after receptive abilities have been accounted for (e.g., Divenyi \& Simon, 1999; Fitzgibbons \& GordonSalant, 1996; Gordon-Salant, 1987; Helfer \& Wilber, 1990; Pichora-Fuller, Schneider, \& Daneman, 1995; Wingfield \& Tun, 2001). Various factors have been proposed to explain the residual effect of age, including deterioration in attentional control (e.g., Hasher, Stoltzfus, Zacks, \& Rypma, 1991; Hasher \& Zacks, 1988; McDowd \& Shaw, 2000; Tun, O'Kane, \& Wingfield, 2002), 
decline of working memory (e.g., Craik, 1994), and a slowdown of cognitive functions (e.g., Salthouse, 1996).

In this study, we investigated the effect of one of these nonsensory factors, attentional control, on speech perception by younger and older listeners. We focused on two facets of attentional control: inhibition of irrelevant information and divided attention. We chose phoneme categorization (Liberman, Harris, Hoffman, \& Griffith, 1957) as our experimental paradigm because, as described below, this paradigm lends itself well to the study of both of those aspects. In a typical phoneme categorization experiment, participants hear syllables one at a time and must decide if the syllable starts with one of two prespecified phonemes (e.g., $g$ vs. $k$ ). The initial phoneme of the syllable is generally manipulated such that its identity is either unambiguous (e.g., a clear $g$ or a clear $k$ ) or ambiguous (e.g., a blend between $g$ and $k$ achieved, e.g., by synthesizing intermediate values of voice onset time, VOT). A key finding is that phoneme categorization is influenced by the perceived lexical status of the syllable (Ganong, 1980; see also Fox, 1984). For example, English listeners tend to report more $g$ responses along a gift-kift continuum and more $k$ responses along a giss-kiss continuum, even though the same set of initial segments is used in both continua. This result suggests that listeners are unable to fully inhibit lexical activation (gift or kiss in this case) when perceiving phonemes. We refer to this finding as the Ganong effect, one of the main dependent variables in the present study.

How can the Ganong effect be used as a probe for the role of attentional control in language processing in older listeners? With respect to the issue of inhibition of irrelevant information, under the hypothesis that older adults suffer from weaker inhibitory control compared to younger adults (Hamm \& Hasher, 1992; Hartman, \& Hasher, 1991; Hasher et al., 1991; Tipper, 1991), they should be particularly poor at inhibiting lexical activation during phoneme categorization, and hence, they should exhibit a greater Ganong effect than younger adults. This is indeed what Baum (2003) found. When older adults were asked to categorize the initial phoneme on a diamond-timond continuum and on a dimingtiming continuum, their propensity to report $d$ in the former and $t$ in the latter was greater than that of younger adults. This lexical bias is also consistent with Sommers' finding that older listeners can less readily suppress the activation of lexical neighbors than younger listeners during spoken-word recognition (Sommers, 1996; Sommers \& Danielson, 1999). Similarly, using eye-tracking methodology and the visual paradigm, Ben-David et al. (2011) showed that, upon hearing a target word (e.g., candle), older adults have more difficulty than younger adults inhibiting eye fixations to the picture of a word sharing its rhyme with the target (e.g., a sandal), suggesting that suppressing lexical competitors becomes more difficult with age. While Sommers' and Ben-David et al.'s results could be interpreted as an age-related weakening of hardwired connectivity within the lexicon rather than a deterioration of attentional control per se (Burke \& Shafto, 2008; see also MacKay \& Burke, 1990), both these data and Baum's confirm the effect of age on lexical activation during speech processing.

It is important to note, however, that the above patterns could also have a sensory origin linked to age-related hearing loss. In particular, Baum's older adults could have favored lexical responses not because they failed to inhibit access to lexical knowledge but simply because they could not distinguish the stimuli on the $d-t$ continuum as well as their younger counterparts. In the present study, we measured the Ganong effect in younger and older adults (as in Baum, 2003) and, importantly, we also measured their ability to discriminate the syllables along the test continua as an index of perceptual acuity. Listeners heard pairs of syllables from the continuum and were asked to report if the initial sounds of the two syllables were the same or different. Thus, the categorization task aimed to capture the listeners' ability to inhibit lexical access whereas the discrimination task aimed to capture their ability to perceive some of the fine acoustic details necessary for the categorization task. Moreover, in an attempt to minimize the contribution of perceptual differences between the two groups, we used a pair of stimuli involving a place-of-articulation contrast $(m-n)$ rather than a time contrast (VOT). This choice was motivated by evidence for increased difficulty with temporal distinctions in older age (e.g., Schneider, Pichora-Fuller, Kowalchuk, \& Lamb, 1994; Strouse, Ashmead, Ohde, \& Grantham, 1998) and, conversely, data showing a relatively small age-related decline in the perception of spectral detail (Ohde \& Abou-Khalil, 2001). Thus, our first question was whether older adults would show a larger Ganong effect compared to younger adults, as predicted by the inhibitory-deficit hypothesis, even when their capacity to discriminate the test stimuli is taken into account.

As mentioned earlier, the second facet of attentional control investigated in this study, alongside inhibitory functions, was divided attention. Testing young adults, Mattys and Wiget (2011) found that the size of the Ganong effect increased when the phoneme-categorization task was performed concurrently with a distracting visual-search task. We will refer to this pattern as lexical drift: a stronger lexical influence on observed behavior under divided attention. This lexical drift was accompanied by poorer syllable discrimination. A question for the present study is whether divided attention will similarly result in a lexical drift and in poorer discrimination in older adults and, if so, whether the cost of divided attention will be the same in both groups or greater in older participants.

\section{Method}

\section{Participants}

All participants were native Dutch speakers drawn from the Max Planck Institute (MPI) for Psycholinguistics participant pool. The younger group consisted of 80 normal-hearing university students (67 female; mean age: 20.6 years; $S D$ : 1.9; age range: 17-27 years) and the older group consisted of 76 listeners aged 60 years and over (51 female; mean age: 68.4 years; $S D$ : 6.7; age range: 60-88 years). Eighty older participants were initially tested, but four of them were removed from the sample because they wore hearing aids in daily life. All participants were paid for their participation.

Hearing sensitivity for the older participants was assessed with a portable Maico ST 25 screening audiometer (air conduction thresholds only; octave frequencies from $250 \mathrm{~Hz}$ to $8 \mathrm{kHz}$ ) in a sound-attenuated booth. Pure-tone average (PTA) thresholds calculated in the participants' better ear were: $.5 \mathrm{~Hz}: 18.5 \mathrm{~dB} \mathrm{HL}(S D=$ 8.0); $1 \mathrm{kHz}: 17.1 \mathrm{~dB}$ HL ( $S D=9.2) ; 2 \mathrm{kHz}: 22.3 \mathrm{~dB}$ HL $(S D=$ 14.1); 4 kHz: $31.1 \mathrm{~dB}$ HL $(S D=19.3)$; 8 kHz: $49.4 \mathrm{~dB} \mathrm{HL}(S D=22.8)$. For reference, normal PTA thresholds are typically below $25 \mathrm{~dB}$ (Clark, 1981). Given that age-related hearing loss is usually con- 
centrated in the higher frequencies, PTA thresholds were also computed separately for low frequencies (better-ear average for .5, 1 , and $2 \mathrm{kHz}$ ) and the high frequencies (better-ear average for 4 and $8 \mathrm{kHz})$. The mean PTA threshold was $18.2 \mathrm{~dB} \mathrm{HL}(S D=8.8)$ for the low frequencies and $36.6 \mathrm{~dB} \mathrm{HL}(S D=18.7)$ for the high frequencies.

\section{Materials}

The stimuli for the categorization and discrimination tasks consisted of three 8-step continua: micht-nicht (nonword-word, where nicht means niece or cousin in English), mist-nist (word-nonword, where mist means mist in English), and a reference nonlexical stem continuum $m i-n i$.

To create the auditory continuum, several renditions of nicht and mist were produced in isolation by a female native speaker of Dutch in a sound-attenuated booth and digitally recorded at 44.1 $\mathrm{kHz}$. The clearest token for each of the two words was selected. Its initial consonant-vowel sequence was then excised using Praat, a software program for the analysis and manipulation of speech signals (Boersma \& Weenink, 2005). The excised [mI] and [nI] sequences were then morphed to create an 11-equally-spaced-step [mI-nI] continuum using STRAIGHT, a computerized tool for speech analysis, modification, and synthesis (Kawahara, MasudaKatsuse, \& de Cheveigné, 1999) running in Matlab. The 11 syllables were subsequently concatenated to the beginning of $/ \chi \mathrm{t} /$ and /st/, yielding an 11-step continuum from micht to nicht (nonwordword) and one from mist to nist (word-nonword). The amplitude of all syllables was set at $70 \mathrm{~dB}$ SPL. The average duration of the three continua was $594 \mathrm{~ms}$ for the micht-nicht continuum, $592 \mathrm{~ms}$ for the mist-nist continuum, and $274 \mathrm{~ms}$ for the base mi-ni continuum.

The 11 syllables on the mi-ni continuum were then piloted to establish the perceptual adequacy of the morphed continuum. The syllables were presented in five blocks, each consisting of 33 items (three repetitions of each syllable). Thus each of the 11 syllables was heard 15 times in total. The task for the participants was to indicate by button press whether the initial sound of the stimulus was $/ \mathrm{m} /$ or $/ \mathrm{n} /$. Each stimulus was presented over headphones 500 ms after trial onset. Seven adults over 60 years of age, who did not participate in the main experiment, took part in the pilot experiment. After analysis of their categorization curves it was decided to create a new continuum using step 4 and step 8 of the initial continuum as endpoints because this region most tightly and symmetrically captured the $\mathrm{m}-\mathrm{n}$ categorization space. Then, following the procedure described above, this restricted region was morphed into a new 8-step continuum. These 8 syllables were concatenated to $/ \chi \mathrm{t} /$, and $/ \mathrm{st} /$, as in the first iteration, to create new micht-nicht and mist-nist continua, which were used in the present study.

The visual arrays used in the divided-attention condition consisted of arrays made of colored shapes. These comprised either two rows and two columns $(2 \times 2$, low load $)$ or six rows and six columns $(6 \times 6$, high load $)$. The distinction between these two degrees of load was not as central to this research as the distinction between no load (focused attention) and load (divided attention), but we included the $2 \times 2$ versus $6 \times 6$ contrast mainly to see if any differences between focused and divided attention were also visible between the two grades of divided attention. Following
Mattys and Wiget (2011), the arrays contained black squares and red triangles, randomly distributed. Half the arrays were targetpresent, that is, they contained a red square, which was the target participants were required to detect. The red square could be anywhere in the array. For the high-load condition, we created a total of 40 different target-absent arrays and 40 different targetpresent arrays. For the low-load condition, we could only create 14 target-absent and 32 target-present arrays due to the limited number of possible permutations. The recycling of arrays in the categorization and the discrimination tasks was kept to a minimum. On the computer monitor, the $2 \times 2$ arrays were $7 \mathrm{~cm}$ by $7 \mathrm{~cm}$ and the $6 \times 6$ arrays were $22 \mathrm{~cm}$ by $22 \mathrm{~cm}$. Each shape in an array was 2.5 $\mathrm{cm}$ by $2.5 \mathrm{~cm}$. The display size was larger than in Mattys and Wiget (2011) to compensate for possible reduced visual acuity in the older participants.

\section{Procedure}

The categorization task always preceded the discrimination task. In both tasks, the focused- and divided-attention conditions were blocked and the order between the two blocks was counterbalanced across participants. Trials in the high-load and low-load conditions of the divided-attention block were always randomized for presentation. Participants were tested individually in a soundattenuated booth. The stimuli were played binaurally over Sennheiser HD 280-13 headphones. In the divided-attention conditions, participants were additionally asked to pay attention to the visual array displayed concurrently with the auditory stimulus, and search for a red square. The visual array was shown on a computer monitor approximately $50 \mathrm{~cm}$ away from the viewer. The experiment lasted approximately 45 minutes.

In the categorization task, the divided-attention block was always immediately preceded by a visual-search task without simultaneous auditory stimuli. The aim of this block was to familiarize the participants with the secondary task they had to perform in the subsequent divided-attention condition. This block contained 60 trials, half of them displaying $2 \times 2$ arrays and the other half $6 \times$ 6 arrays, mixed and randomized. Furthermore, to ensure that the older participants fully understood the instructions in the phoneme-categorization task, they received two short practice sessions (four trials), one preceding the focused-attention block and one preceding the divided-attention block.

In the focused-attention block of the categorization task, participants heard each syllable of the three continua one at a time, for a total of 24 syllables. These were played in a random order. On each trial, the syllable was immediately followed by the presentation of the letters $m$ and $n$ in the left and right bottom of the screen, respectively. Using a button box, participants were asked to indicate whether the initial sound of the stimulus was $/ \mathrm{m} / \mathrm{or} / \mathrm{n} /$. They were explicitly encouraged to focus on the initial sound and ignore the meaning of the syllable. Participants had up to $10 \mathrm{~s}$ to press a button. After a button press or the 10-s period, there was a 2-s intertrial interval, after which the next syllable was played.

In the divided-attention block of the categorization task, participants heard the 24 syllables twice: Once concurrently with the presentation of a $2 \times 2$ array (low load) and once with the presentation of a $6 \times 6$ array (high load), for a total of 48 trials. These were mixed and presented randomly. The assignment of the arrays to the syllables was semirandom: If a syllable was played 
with a target-present array in the low-load condition, that same syllable was played with a target-absent array in the high-load condition, and vice versa. The timing of each trial was comparable to that of the focused-attention condition, except that a visual array was displayed on the computer monitor while the auditory syllable played. To keep timing constant for all syllables, the array was displayed for $605 \mathrm{~ms}$, the duration of the longest syllable. This was immediately followed by the display of the $m$ and $n$ letters. Again, participants had up to $10 \mathrm{~s}$ to press a button, after which the following message appeared on the screen: "nee .... rode vierkant? ... . ja" (no .... red square? ... . yes). Participants had up to $10 \mathrm{~s}$ from the onset of the message to indicate the absence (left button) or presence (right button) of the red square. After button press or the 10-s wait, there was a 2 -s intertrial interval, after which the next syllable and visual array were presented.

In the discrimination task, each auditory stimulus consisted of a pair of syllables. The syllables within a pair were always identical except for the first phoneme, which could be either the same phoneme or different phonemes $(/ \mathrm{m} / \mathrm{vs} . / \mathrm{n} /)$. The syllables were those used in the categorization task, excluding the endpoint syllables (1 and 8), thus leaving syllables 2, 3, 4, 5, 6, and 7 for each of the three continua. Within each continuum, the test pairs were $2-4,3-5,4-6$, and 5-7. Thus, the syllables within each pair were one syllable away from each other. For convenience, these four test pairs were renamed step 1, step 2, step 3, step 4, respectively. Discrimination for each step involved testing two "different" pairs and two "same" pairs. For example, for step 2, the "different" pairs were 2-4 and 4-2 (reverse order of presentation) and the "same" pairs were 2-2 and 4-4. Steps 1 and 4 tested discrimination near the end points of the continuum whereas steps 2 and 3 tested discrimination near the middle of the continuum. Because some syllable pairings were used for more than one step (4-4 was used for both step 1 and step 3; 5-5 was used for both step 2 and step 4), the number of pairs per continuum was 14 (42 in total).

In the divided-attention block of the discrimination task, all the pairs described above were played under low load $(2 \times 2$ arrays $)$ and high load ( $6 \times 6$ arrays), for a total of 84 pairs presented randomly. Again, the assignment of the arrays to the pairs was semirandom: If a pair was played with a target-present array in the low-load condition, that same pair was played with a target-absent array in the high-load condition, and vice versa.

The timing of each trial in the discrimination task was the same as in the categorization task, except that the prompt displayed on the computer screen for the discrimination task was: "verschillend ... beginklanken? ... gelijk" (different ... initial sounds? ... same). The two syllables of a stimulus pair were separated by 500 ms. Participants were asked to decide if the initial sound of the two syllables was the same or different using the left button for different and the right button for same. In the divided-attention condition, the visual array was displayed for the same duration as in the categorization task ( $605 \mathrm{~ms})$; the onset of the visual array was aligned with the onset of the first syllable.

\section{Results}

\section{Entire Participant Sample}

Phoneme categorization. The phoneme categorization curves for the focused- and divided-attention conditions are displayed in
Figures 1A-B for the younger adults $(n=80)$ and 1C-D for the older adults $(n=76)$. The divided-attention condition displayed in the figures is an average of the low $(2 \times 2$ arrays $)$ and high $(6 \times$ 6 arrays) conditions, since these did not differ significantly, as described later. The main finding was that the Ganong effect, calculated as the difference between the _icht and_ist conditions (i.e., the average across the eight steps of the _icht continuum minus the average across the eight steps of the _ist continuum), was larger for the older than the younger group and under divided than focused attention. There was no evidence that the increased Ganong effect under divided attention (i.e., the lexical drift) was more pronounced for the older than younger listeners.

The analysis of variance that led to these conclusions was performed on the participants' Ganong effect as a function of Attention (focused, divided-low, divided-high) and Age (younger, older). Although the responses in the $\_i$ context are displayed in Figure 1, they were not included in the analyses because they are not of direct relevance to the measurement of the Ganong effect. The size of the Ganong effect was influenced by both Attention, $F(2,306)=7.81, p<.001$, and Age, $F(1,153)=66.48, p<.001$. These two variables did not interact, $F(2,306)<1$. The Attention effect showed that both the low and high divided-attention conditions produced a greater Ganong effect than the focused-attention condition, $F(1,153)=11.68, p=.001$, and $F(1,153)=7.88, p<$ .01 , respectively. The two divided-attention conditions did not differ from each other, $F(1,153)<1$.

An analysis of variance of the visual-search task showed better detection of the visual target in the low- than high-load condition $(93 \%$ vs. $82 \%, F(1,153)=157.55, p<.001)$, and marginally better performance by younger than older listeners ( $90 \%$ vs. $85 \%$, $F(1,153)=3.40, p=.07)$. A significant interaction between Load Level and Age, $F(1,153)=55.20, p<.001$, revealed that the younger and older participants were equally good in the low-load condition (92\% vs. 93\%, $F(1,153)<1$ ), but the older participants were poorer than the younger ones in the high-load condition $(88 \%$ vs. $77 \%, F(1,153)=15.43, p<.001)$. Thus, even though the load difference in the divided-attention condition was effective-and especially so for the older adults-it had no impact on the phoneme categorization task; only the focused versus divided contrast did.

Phoneme discrimination. Discrimination accuracy was calculated for each participant and for each cell of the design as the percentage of correctly responded-to pairs on a quadruplet-of-pairs basis (e.g., syllable pairs 2-4, 4-2, 2-2, 4-4). We chose this method over $d^{\prime}$ and Beta from Signal Detection Theory (Green \& Swets, 1966) because of the large proportion of $0 \%$ and $100 \%$ values among the cells of the design - a consequence of using a single item-which is problematic for calculating statistics derived from $z$ scores. The results are shown in Figures 2A for the younger adults and Figure 2B for the older adults. As in the phoneme categorization figures, the divided-attention condition was an average of the low-load $(2 \times 2$ arrays $)$ and high-load $(6 \times 6$ arrays $)$ conditions, as these were not found to differ significantly. Discrimination was averaged across the three contexts (_icht, _ist, and _i) because this variable was neither of theoretical interest in this analysis nor did it significantly interact with any of the main patterns.

An analysis of variance on the discrimination scores was performed on Step (1, 2, 3, 4), Attention (focused, divided-low, 
A. Younger: Focused attention

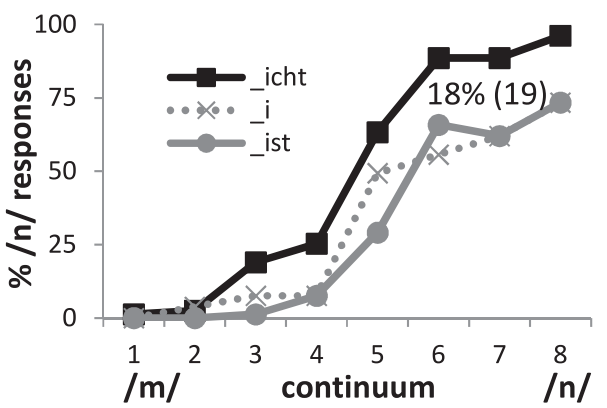

C. Older: Focused attention

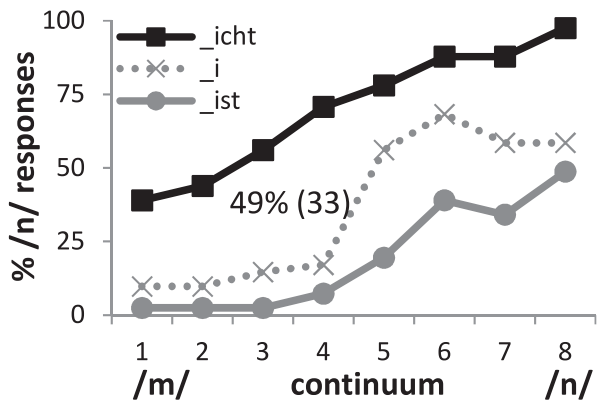

B. Younger: Divided attention

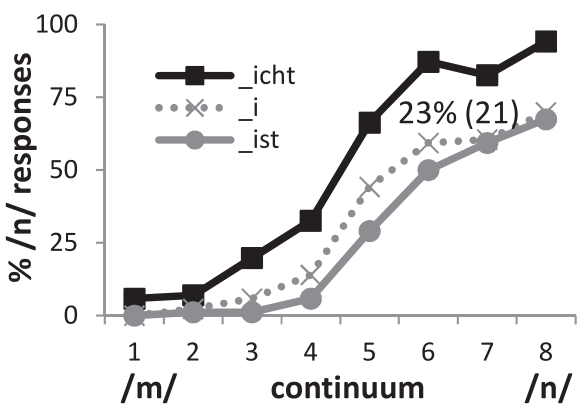

D. Older: Divided attention

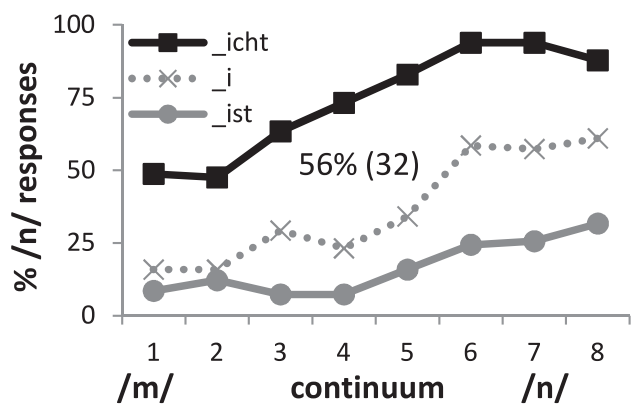

Figure 1. Percentage of $/ \mathrm{m} /$ responses for the micht-nicht, mi-ni, and mist-nist continua in the phoneme categorization task as a function of Age (younger, older) and Attention (focused, divided). The Ganong effect, shown as a percentage value in the middle of each figure (standard deviation in parentheses), was calculated as the difference between the $/ \mathrm{n} /$ responses in the_icht condition and the $/ \mathrm{n} /$ responses in the _ist condition averaged across the eight steps of the continuum.

divided-high), and Age (younger, older). A main effect of Step, $F(3,918)=44.55, p<.001$, showed that discrimination was better for the two middle steps than for the two far-end steps, which is in keeping with evidence for better discrimination of speech sounds across phoneme boundaries than within phoneme categories (Liberman et al., 1957; Repp, 1984). All pairwise comparisons over the Step factor reached $p<.005$ (without correction), except for the comparison between step 1 and step $4, F(1$,
$153)=3.45, p=.06$. While a main effect of Age indicated that discrimination was better in younger than older listeners, $F(1$, $153)=8.98, p<.005$, this was not the case for all four steps, as shown by a Step-by-Age interaction, $F(3,459)=6.56, p<.001$. The Age effect was reliable at step $2, F(1,153)=8.73, p<.005$ and step $3, F(1,153)=10.74, p=.001$, but not at step $1(F(1$, $153)<1)$ or step $4, F(1,153)=1.65, p=.20$, suggesting that, in both age groups, discrimination was at or near chance level for

\section{A. Younger adults}

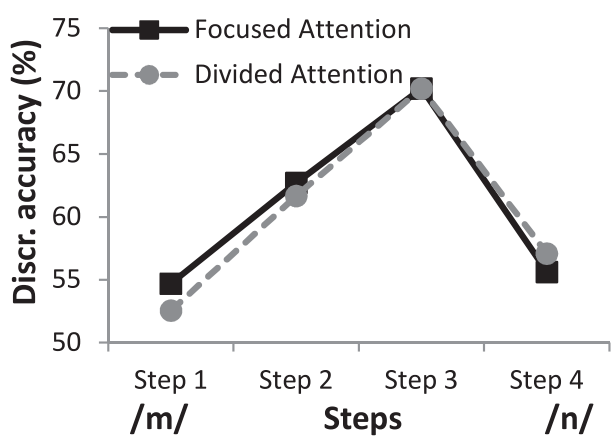

B. Older adults

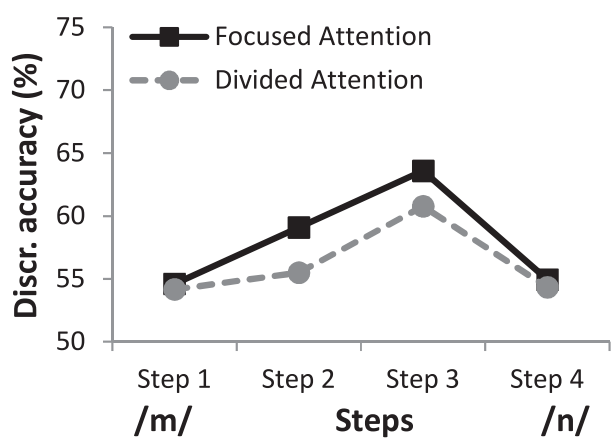

Figure 2. Percentage of correct discrimination between syllables along the $/ \mathrm{m}-/ \mathrm{m}$ continuum as a function of Age (younger, older) and Attention (focused, divided). Results are averaged across the _icht, _ $i$, and _ ist contexts. 
tokens within phonemic categories. There was no main effect of Attention, $F(2,306)=1.81, p=.17$. Finally, although not significant, a marginal interaction between Attention and Age, $F(2$, $306)=2.56, p=.08$, hinted that Attention affected the older group, $F(2,148)=4.31, p=.01$, but not the younger group, $F(2$, $158)<1$. The older group showed better discrimination in the focused than in the divided-low condition, $F(1,74)=8.26, p=$ .005 , but neither of the other two pairwise comparisons (focused vs. divided-high, divided-low vs. divided-high) reached significance. However, an analysis (of the older group) restricted to steps 2 and 3, that is, those steps that showed the best discrimination potential, showed a clear contrast between focused attention and both divided-low and divided-high attention, $F(1,74)=7.79, p<$ .01 , and $F(1,74)=7.80, p<.01$, respectively, and no difference between divided-low and divided-high, $F(1,74)<1$. For the younger group, none of these comparisons reached significance (all $p \mathrm{~s}>$.15).

As for the performance on the visual-search task, the younger participants outperformed the older ones $(95 \%$ vs. $90 \%, F(1$, $153)=18.12, p<.001)$ and target detection was worse in the high-load than the low-load condition $(96 \%$ vs. $89 \%, F(1,153)=$ 121.76, $p<.001)$. However, an Age-by-Load interaction, $F(1$, $153)=71.21, p<.001$, showed that the older participants were more affected by the load difference than the younger participants (older: $F(1,74)=125.59, p<.001$; younger: $F(1,79)=6.14$, $p=.01)$. Younger and older participants were equally good in the low-load condition $(96 \%$ vs. $96 \%, F(1,153)<1)$, but the younger participants did better than the older ones in the high-load condition $(94 \%$ vs. $84 \%, F(1,153)=49.36, p<.001)$. There were no main effects of Step or interactions involving Step. Thus, again, even though the high-load condition was more difficult than the low-load condition, the difference had no notable impact on discrimination; only the presence versus absence of the secondary task did.

Summary and discussion. The younger and older adults exhibited contrasting patterns of results in both the categorization and the discrimination tasks. The older adults showed a greater Ganong effect and poorer discrimination than the younger adults. The former result replicates Baum's (2003) finding and the latter is in line with a vast literature on age-related reduced hearing sensitivity. The larger Ganong effect for the older listeners is consistent with the hypothesis that older adults have more difficulties than younger ones inhibiting irrelevant information (e.g., Hasher et al., 1991), in this case, lexical knowledge (Sommers, 1996). However, because the older adults had poorer discrimination scores, it is possible that their larger Ganong effect was merely a byproduct of sensory decline rather than a genuine deficiency with lexical inhibition. Since this issue was one of the main research questions at the outset of this study, it will be addressed with further analyses in the next section.

The addition of a secondary task had mixed consequences. First, whatever effect the secondary task had, it was mainly due to the contrast between focused and divided attention. The degree of difficulty of the secondary task $(2 \times 2$ vs. $6 \times 6$ visual arrays $)$ was itself inconsequential. This is not to say that cognitive effort was comparable in the low-load $(2 \times 2)$ and high-load $(6 \times 6)$ conditions, as target detection was generally higher in the former than the latter, but its impact on phoneme categorization and discrimination was small.
Divided attention had no effect on discrimination in the younger group but there was some indication that it did in the older group. The lack of an effect in the younger group is somewhat surprising in light of Mattys and Wiget's (2011) data. It is possible that the visual task in the present experiment was less taxing than that in Mattys and Wiget. Visual target detection was indeed higher for the younger adults in this study (94\%, high load) than for the younger adults in Mattys and Wiget's (84\%). The larger visual display in this study could have contributed to this difference. Discrimination, too, was higher here. The difference could be due to the distinct phonetic contrasts (/m-n/ vs. /g-k/) and the way they were created (resynthesized vs. edited). Regardless, the fact that the older adults' discrimination performance was more detrimentally affected by divided attention than that of the younger adults (see also Humes, Lee, \& Coughlin, 2006) can be interpreted as another instance of decreased attentional control (e.g., Craik \& Byrd, 1982; Wright, 1981) alongside the decrease in inhibitory capacity suggested by the Ganong data.

As for the effect of divided attention on phoneme categorization, both age groups showed a larger Ganong effect under divided than focused attention. This replicates Mattys and Wiget's (2011) finding of a lexical drift under load. Divided attention seems to divert younger and older listeners' attention away from the acoustic detail of the speech signal, causing both groups to fall back on alternative sources of information to perform the categorization task, in this case lexical knowledge. However, the lexical drift was the same magnitude in both age groups. This could mean that the two groups did not fundamentally differ in how they responded to the dual task. This, in turn, could imply that the resources needed to cope with divided attention are distinct from those needed to inhibit lexical activation, with the former relatively insensitive to aging and the latter more so. This interpretation should be taken with caution however, because it is possible that the already large Ganong effect in the older participants under focused attention made it difficult for divided attention to yield a measurable added Ganong effect compared to that in the younger group.

\section{Matched Participant Subsamples}

The main question at the outset of this study was whether age-related speech-processing difficulties could be partly accounted for by older adults' impoverished ability to control attention, and particularly attentional allocation to acoustic versus lexical information. Our older participants' larger Ganong effect (weakened inhibition) and greater sensitivity to dual-tasking during phoneme discrimination are consistent with this possibility, but, as discussed earlier, these patterns could also be the byproduct of poorer phonetic perception, as suggested by the older participants' lower discrimination scores. To tease apart these two alternatives, we reran our main analyses on subsamples of younger and older participants matched pairwise on their discrimination score in the focused-attention condition. The discrimination score used as a basis for matching was the average of steps 2 and 3 because these were found to most clearly distinguish the two age groups. Participants were ranked on this score within their own age group. Participants with scores at chance level or below $(\leq 50 \%)$ were removed. The remaining participants were matched within each discrimination accuracy band across age groups. For example, among the 13 older participants who scored $54 \%$, only three were 
kept in order to match the number of younger participants with the same score. The three older participants were chosen at random. We followed this procedure for each discrimination band. The two resulting subsamples contained 43 participants each. Their distributions of scores were identical (see Figure 3). Discrimination and categorization results for these subsamples are shown in Figures $4 \mathrm{~A}$ for the younger group and 4B for the older group. For concision, the $i$ condition is not shown.

In the following analyses, the low and high divided-attention conditions were collapsed because they were not found to significantly differ in the previous analyses. As a direct consequence of subsampling, Age was not significant in the discrimination analysis, $F(1,42)=1.38, p=.25$. When considering only the most discriminable steps (steps 2 and 3), an interaction between Age and Attention, $F(1,42)=4.42, p<.05$, showed that divided attention impoverished discrimination in the older participants, $F(1,42)=$ $15.11, p<.001$, but not in the younger participants, $F(1,42)<1$. Thus, the age-specific effect of divided attention on discrimination noted in the full-sample analysis (although only marginally significant) firmed up when both groups were equated on their discrimination accuracy under focused attention.

More importantly, with respect to the categorization task, the effect of Age on the Ganong effect was still present, $F(1,42)=$ 31.62, $p<.001:$ As in the full-sample analysis, older adults showed a larger Ganong effect than younger adults. The Attention effect remained as well, $F(1,42)=4.43, p<.05$, with a larger Ganong effect under divided than focused attention. As before, Age and Attention did not interact, $F(1,42)<1$ : There was no evidence that the lexical drift under divided attention was larger in the older than younger participants. In sum, even when phoneme discrimination was controlled for, we found that: (1) The ability to inhibit lexical activation decreased with age, (2) Divided attention impaired phoneme discrimination more in older than younger listeners, and (3) Divided attention increased reliance on lexical knowledge (larger Ganong effect) for both younger and older adults. Thus, to answer our initial research question, age-related speech-processing difficulties can be partly accounted for by an impoverished ability to control attention in older age.

\section{Role of Hearing Sensitivity and Age on Performance in Older Adults}

In an attempt to further investigate the role of hearing sensitivity in age-related speech-perception difficulties among our older participants, we used their audiometric data as a predictor of their phonetic categorization and discrimination scores. We restricted these analyses to the older group because variation in hearing sensitivity is known to be greater among older than younger adults and because the within-group age range was wider in the former (20 years) than the latter (10 years). We considered pure-tone average (PTA) threshold in the better ear separately for low frequencies (averaged over .5, 1, and $2 \mathrm{kHz}$ ) and high frequencies (averaged over 4 and $8 \mathrm{kHz}$ ). Because the predictive power of the low frequencies turned out to be consistent with, but generally less pronounced than that of the high frequencies, only the results for the high frequencies are reported.

Age was also entered as a predictor to investigate whether there are enduring effects of age on speech processing even after lowlevel receptive abilities have been accounted for. We were particularly interested in whether hearing sensitivity and age modified the size of the Ganong effect, the increase of the Ganong effect under divided attention compared to focused attention, that is, the lexical drift, discrimination accuracy under focused attention, and the decrease in discrimination accuracy under divided attention compared to focused attention. Table 1 shows pairwise correlations between age, hearing sensitivity, and: (1) the size of the Ganong effect under focused attention, (2) the size of the Ganong effect under divided attention, (3) discrimination accuracy under
A. Full samples

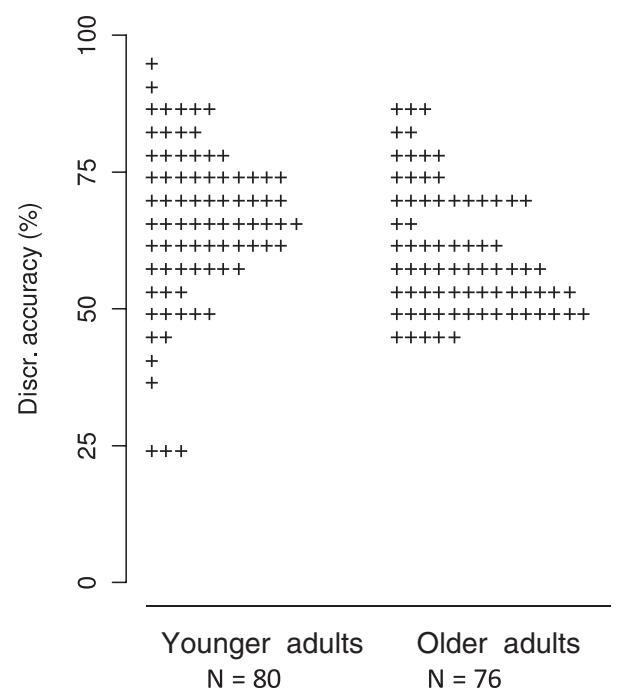

\section{B. Matched sub-samples}

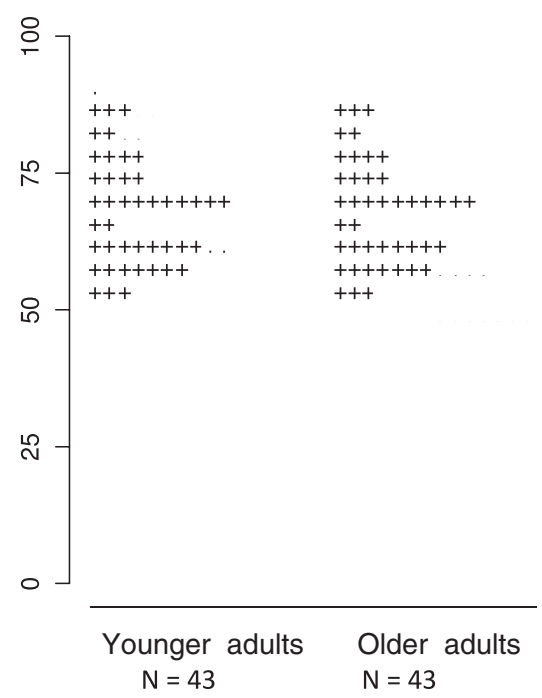

Figure 3. Distributions of discrimination scores under focused attention for younger and older participants. A. When all participants are considered. B. With subsamples of participants paired on their discrimination scores. 
A. Younger Adults
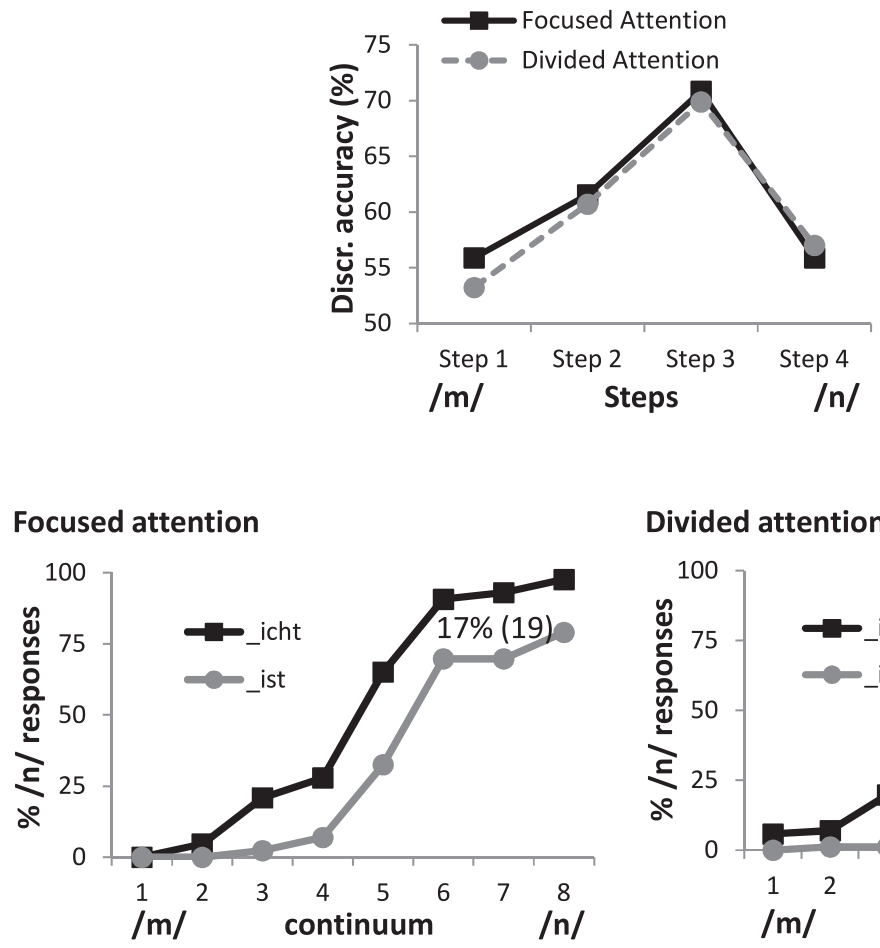

Divided attention

B. Older Adults
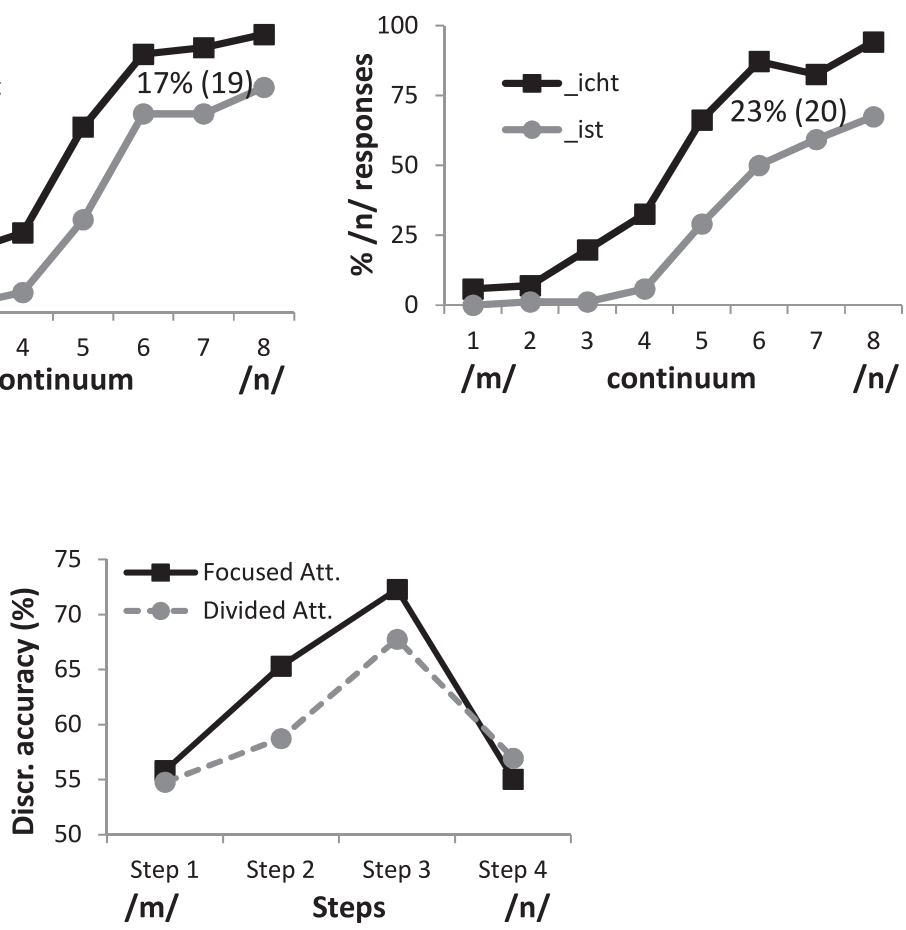

Focused attention

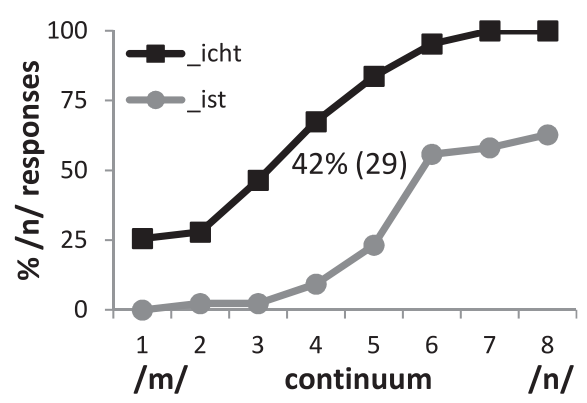

Divided attention

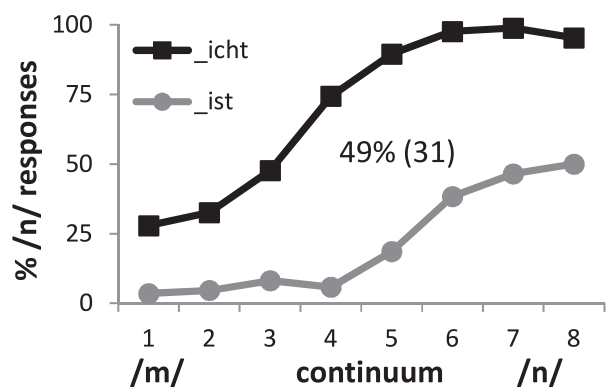

Figure 4. Discrimination (top panel) and phoneme categorization performance (bottom panels) under focused and divided attention for subsamples of younger (A) and older participants (B) paired on their discrimination performance under focused attention (calculated on the average of Steps 2 and 3). 
Table 1

Correlation Matrix Between Main Variables for the Older Adults $(\mathrm{N}=76)$

\begin{tabular}{lcccccc}
\hline & Age & $\begin{array}{c}\text { Hearing low } \\
\text { freq. }\end{array}$ & $\begin{array}{c}\text { Hearing high } \\
\text { freq. }\end{array}$ & $\begin{array}{c}\text { Ganong } \\
\text { focused }\end{array}$ & $\begin{array}{c}\text { Ganong } \\
\text { divided }\end{array}$ & $\begin{array}{c}\text { Discrim. } \\
\text { focused }\end{array}$ \\
\hline Hearing low freq. & $.48^{* * * *}$ & & & & & \\
Hearing high freq. & $.62^{* * * *}$ & $.55^{* * *}$ & & & \\
Ganong focused & .11 & .14 & .14 & & \\
Ganong divided & -.03 & .11 & .19 & $.69^{* * * *}$ & & \\
Discrim. focused & $-.38^{* * *}$ & $-.29^{* *}$ & $-.43^{* * * *}$ & $-.30^{* * *}$ & $-.38^{* * *}$ & \\
Discrim. divided & $-.37^{* *}$ & $-.30^{* *}$ & $-.41^{* * *}$ & $-.36^{* *}$ & $-.39^{* *}$ & $.73^{* * *}$ \\
\hline
\end{tabular}

Note. $\quad$ Hearing low freq. $=$ Pure-tone average $(\mathrm{PTA})$ threshold in better ear averaged over $.5,1$, and $2 \mathrm{kHz}$; Hearing high freq. $=$ Pure-tone average (PTA) threshold in better ear averaged over 4 and $8 \mathrm{kHz}$; Ganong focused $=$ size of the Ganong effect under focused attention; Ganong divided $=$ size of the Ganong effect under divided attention; Discrim. focused $=$ Discrimination accuracy under focused attention; Discrim. divided $=$ Discrimination accuracy under divided attention.

$*<.05$. $^{* *}<.01$. $^{* * * *}<.001$.

focused attention, and (4) discrimination accuracy under divided attention. In the regression analyses that follow, we first present the discrimination analyses because discrimination is a potentially informative predictor of categorization performance.

Phoneme discrimination. Age and hearing sensitivity (restricted to the high frequencies throughout the analyses), considered together, significantly predicted discrimination under focused attention, adjusted $R^{2}=.19, F(2,73)=9.61, p<.001$. However, the unique contribution of each of factor over and above the other one was only significant for hearing sensitivity, standardized $\beta=-.31, p<.05$. Age: standardized $\beta=-.19, p=.16$. Thus, the stronger predictor of phoneme discrimination under focused attention was hearing sensitivity, not age per se.

We then assessed whether age and hearing sensitivity predicted the drop in discrimination between focused and divided attention using a residualized change analysis. The drop in discrimination was instantiated as the unstandardized residuals of a regression analysis between discrimination under focused attention (the independent variable) and discrimination under divided attention (the dependent variable). Age and hearing sensitivity, which were then used as predictors of these residual values, did not significantly improve data fit, adjusted $R^{2}=.00, F(2,72)<1$. Age, standardized $\beta=-.07, p=.64$. Hearing sensitivity: standardized $\beta=-.10, p=.52$. Thus, neither age nor hearing sensitivity had a significant effect on the deterioration of discrimination accuracy under divided attention compared to focused attention.

Phoneme categorization. We first considered the influence of age and hearing sensitivity on the size of the Ganong effect under focused attention. Because the size of the Ganong effect under focused attention was negatively correlated with discrimination under focused attention (see Table 1), we built an initial model with discrimination under focused attention as the sole predictor of the Ganong effect under focused attention to ensure that any subsequent improvement of the model with age and hearing sensitivity could not be explained by individual differences in discrimination accuracy. As expected, a larger Ganong effect under focused attention was associated with poorer discrimination, adjusted $R^{2}=.08, F(1,74)=7.44, p<.01 ;$ standardized $\beta=-.30$, $p<.01$. Adding age and hearing sensitivity did not improve this model, adjusted $R^{2}$ change $=.00, F(2,72)<1$. Age, standardized $\beta=-.02, p=.88$. Hearing sensitivity: standardized $\beta=.02, p=$
.87. Thus, age and hearing sensitivity did not have a measurable effect on the size of the Ganong effect beyond the effect they had on discrimination.

Finally, we examined the effect of age and hearing sensitivity on the increase of the Ganong effect under divided attention compared to focused attention, that is, the size of the lexical drift. Following the logic of the discrimination analysis, the lexical drift was instantiated as the unstandardized residuals of a regression between the Ganong effect under focused attention (the independent variable) and the Ganong effect under divided attention (the dependent variable). We then built a model that included only discrimination under focused attention as a predictor of the residual values in order to isolate basic discriminability from the contribution to the lexical drift of age and hearing sensitivity. Discrimination under focused attention was found to significantly predict the lexical drift, adjusted $R^{2}=.04, F(1,74)=4.31, p<$ .05 ; standardized $\beta=-.08, p=.47$. Participants with poor discrimination scores showed a larger lexical drift, which is in line with group analysis. Adding age and hearing sensitivity improved this model, adjusted $R^{2}$ change $=.11, F(2,72)=4.75, p=.01$. The unique contribution of each of the two factors over and above the other one was significant for age, standardized $\beta=-.43, p<$ .005 , but it only approached significance for hearing sensitivity, standardized $\beta=.27, p=.06$. Thus, once individual variability in discrimination and hearing sensitivity was accounted for, the oldest members of the older group showed a smaller lexical drift.

In summary, within the older group, the increase of the Ganong effect under divided attention compared to focused attention (the lexical drift) was not significantly affected by discrimination ability and was smaller among the oldest participants. The unexpected valence of the age effect seems to have originated from the oldest participants within the group (four individuals 82 years and above), as the pattern disappeared when these individuals were removed from the analysis (standardized $\beta=-.01, p=.13$ ). Thus, a question for future research concerns the extent to which the present findings generalize to a category of individuals that has come to be called the "oldest-old" (Suzman, Willis, \& Manton, 1992; for a review, see von Gunten, Ebbing, Imhof, Giannakopoulos, \& Kövari, 2010). 


\section{General Discussion}

In this study, we investigated the extent to which older listeners' documented difficulties with language processing can attributed to a decline in attentional control independent of age-related deficit in phonetic perception. The two facets of attentional control we examined were inhibition of irrelevant information and divided attention.

Following the inhibitory-deficit hypothesis (e.g., Hamm \& Hasher, 1992; Hartman, \& Hasher, 1991; Hasher et al., 1991), whereby age-related decline in language processing is thought to be linked to a decreased ability to inhibit irrelevant stimuli, we hypothesized that older adults would show poorer lexical inhibition during phoneme categorization (i.e., a larger Ganong effect) than younger adults. This is indeed what we found. This inhibitory contrast was not mere compensation for poorer hearing sensitivity in the older adults since the effect remained when both age groups were equated on their phoneme-discrimination performance. It should be noted that, in addition to weaker lexical inhibition, the larger Ganong effect could also reflect greater robustness of lexical representations in the older participants. With many years of additional exposure to language, the accumulated frequency of lexical representations could bias the relative weights between lexical and sublexical information toward heavier reliance on the lexicon, as suggested by Spieler and Balota (2000) and by studies showing relative stability, if not strengthening, of lexical knowledge in older adults (e.g., Kemper \& Sumner, 2001). Thus, older adults might find it harder to disengage their attention from lexical knowledge for the purpose of what is, after all, a rather artificial task. But it does not mean that they would be unable to attend to sublexical detail if doing so had a more function goal; for example, learning new words. In any case, however, it is clear that the younger and older adults behaved in radically different ways in terms of weighing lexical and sublexical information, and that the pattern could not be entirely accounted for by differences in perceptual sensitivity between the two groups.

With respect to divided attention, the results were mixed. While divided attention increased the Ganong effect in both age groups (as already shown for younger adults by Mattys \& Wiget, 2011), the size of this lexical drift was not significantly larger in the older group. However, divided attention was more detrimental to older than younger adults in the discrimination task. As before, these patterns were not reliably affected by whether or not the two groups were matched on their discrimination scores.

None of the analyses revealed differences between the low and high grades of divided attention $(2 \times 2$ vs. $6 \times 6$ arrays). This could be an indication that the cost incurred by dividing attention is rather categorical, at least as far as its impact on the present tasks is concerned. This would be consistent with Verhaeghen, Steitz, Sliwinski, and Cerella's (2003) assertion that divided attention is not reducible to task complexity, a claim made by McDowd and Craik (1988), and that divided attention engages a qualitatively distinct process only marginally sensitive to gradation (see, e.g., Hartley, 2001). The lack of a difference between the two levels of load also casts doubt on the notion that resources are graded and quantifiable (see Navon, 1984, and Navon \& Gopher, 1979, for a detailed analysis). Had it been the case, a decrease of such resources in older adults should have manifested itself as a heightened sensitivity to the difference between the two levels of load, which was not found. This conclusion should be interpreted with caution, however, because Mattys and Wiget (2011) previously found some evidence for graded effects of array size on the Ganong effect for younger listeners. Whether a greater contrast between the low- and high-load conditions (e.g., $8 \times 8$ instead of $6 \times 6$ ) would lead to measurable effects on the speech tasks is unclear at this stage.

The older listeners' less effective inhibition of lexical information can be considered from both domain-general and languagespecific perspectives. From a domain-general perspective, the effect could arise from the task demands associated with ignoring any type of irrelevant information. On that account, our increased Ganong effect in older participants would be cognitively indistinguishable from the age-related cost of performing a speech task while ignoring the speaker's voice (Yonan \& Sommers, 2000) or background noise (e.g., Pichora-Fuller \& Souza, 2003). From a language-specific perspective, our effect could be specifically related to inhibitory mechanisms inside the lexicon rather than inhibition in general. For instance, within existing wordrecognition models, failure to inhibit lexical activation could be implemented in two ways. In interactive-activation models (e.g., TRACE; McClelland \& Elman, 1986), an excessive intervention of lexical knowledge during phoneme categorization could happen if the top-down connections between the lexical and phoneme layers failed to be inhibited for the purpose of the task. Sommers (1996; Sommers \& Danielson, 1999) had already highlighted age-related deficits associated with lateral inhibition within the lexicon (larger neighborhood-competition effects in older adults). Our results suggest that such deficits may extend to the top-down connections between the lexicon and phonemic representations. In contrast, a feed-forward model such as Merge (Norris, McQueen, \& Cutler, 2000), which does not have top-down connections between lexical and phonemic representations, could account for our results by a failure to inhibit the contribution of lexical activation to the postlexical phoneme-decision layer. As mentioned earlier, whether this effect would be partly under the listeners' control is difficult to establish from the present data-strategic control would be more readily accommodated by Merge than TRACE. However, the net result in both implementations would be an overreliance on lexical knowledge during phoneme categorization, as observed in our older group.

Greater decline in attentional control over speech perception in older adults was also noticed under divided attention. Phonemediscrimination scores were more detrimentally affected by the competing visual task in the older than younger listeners. This disadvantage remained even when discrimination under focused attention was equated between the two groups. Whether this is a genuine instance of age-related attentional deficit (e.g., Hartley \& Little, 1999; McDowd \& Shaw, 2000; Verhaeghen et al., 2003) or merely heightened sensitivity to task complexity (e.g., McDowd \& Craik, 1988) is unclear. However, either interpretation is in contradiction with a strict sensory-deficit hypothesis, which would have predicted a minimal effect of divided attention on discrimination once baseline discrimination accuracy was controlled for. Interestingly, a sensory-deficit interpretation, or a version thereof, might be retained if we adopt Mattys and Wiget's (2011) view that divided attention impairs sensory processing by diverting listeners' attention from the acoustic detail of the speech signal. Here, older adults would show greater sensitivity to divided attention not so 
much because of a deterioration of attentional control functions, but because their auditory abilities would be more easily challenged by divided attention. Equating discrimination levels under focused attention would erase the exterior manifestation of the older listeners' auditory deficit, but not their intrinsically lower tolerance to signal degradation. This notion of auditory "fragility" in older listeners is consistent with findings that older adults can differ from younger ones not only in their absolute auditory thresholds but also in how they process speech above such thresholds (see Humes \& Dubno, 2010, for a review). It is also consistent with data showing the erosion of phonological representations in long-term memory subsequent to prolonged auditory deficit (e.g., Rönnberg et al., 2011), as is likely to be the case in older adults.

With respect to the effect of divided attention on phoneme categorization, we found that both younger and older listeners had greater difficulty inhibiting lexical activation under divided than focused attention, consistent with the lexical-drift pattern described by Mattys and Wiget (2011) for young listeners. However, there was no evidence that this lexical drift was greater in the older than younger group. The lack of an age difference is difficult to interpret as it could be due to a ceiling effect caused by the already large Ganong effect under focused attention in the older group. If the results are to be interpreted at face value, however, they suggest that whatever process is responsible for the age-related increased Ganong effect in the focused condition is distinct from that responsible for the increased Ganong effect under divided attention. Thus, on this account, the resources needed for lexical inhibition and those needed for handling divided attention would support two different facets of attentional control, and these resources would be differentially affected by age: Selectively inhibiting lexical activation would be harder in older than younger listeners, but doing so while paying attention to another stimulus would not increase that deficit.

In conclusion, this study shows that, while a decline in hearing sensitivity is an important contributor to age-related changes in phoneme perception, a strict sensory-deficit approach is not sufficient to account for the present data. We have proposed two ways of addressing this limitation. One solution is to consider deterioration in attentional control, and specifically inhibitory control, as an additional age-related deficiency alongside hearing loss. Our results can then be accommodated by either a domain-general (e.g., Hasher \& Zacks, 1988) or a language-specific (e.g., Sommers, 1996) implementation of inhibition deficit. The other solution is to accept a broader conceptualization of the sensory-deficit approach, whereby sensory fragility (encompassing both threshold and suprathreshold performance) would be responsible for language-processing decline in older adults. In this conceptualization, even hearing loss unnoticeable in optimal circumstances would constitute a sensitive ground for adverse listening conditions, whether these are perceptual (e.g., background noise) or cognitive (e.g., divided attention).

\section{References}

Baum, S. R. (2003). Age differences in the influence of metrical structure on phonetic identification. Speech Communication, 39, 231-242. doi: 10.1016/S0167-6393(02)00028-6

Ben-David, B. M., Chambers, C. G., Daneman, M., Pichora-Fuller, M. K., Reingold, E. M., \& Schneider, B. A. (2011). Effects of aging and noise on real-time spoken word recognition: Evidence from eye movements.
Journal of Speech, Language, and Hearing Research, 54, 243-262. doi:10.1044/1092-4388(2010/09-0233)

Boersma, P., \& Weenink, D. (2005). Praat. Doing phonetics by computer (Version 5.1) [Software]. Amsterdam, The Netherlands: Phonetic Sciences, University of Amsterdam.

Burke, D. M., \& Shafto, M. A. (2008). Language and aging. In T. A. Salthouse \& F. I. M. Craik (Eds.), The handbook of aging and cognition (pp. 373-443). New York, NY: Psychology Press.

Clark, J. G. (1981). Uses and abuses of hearing loss classification. ASHA, 23, 493-500.

Craik, F. I. M. (1994). Memory changes in normal aging. Current Directions in Psychological Science, 3, 155-158. doi:10.1111/1467-8721 .ep10770653

Craik, F. I. M., \& Byrd, M. (1982). Aging and cognitive deficits: The role of attentional resources. In F. I. M. Craik \& S. E. Trehub (Eds.), Aging and cognitive processes (pp. 191-211). New York, NY: Plenum Press. doi:10.1007/978-1-4684-4178-9_11

Divenyi, P. L., \& Simon, H. J. (1999). Hearing in aging: Issues old and young. Current Opinion in Otolaryngology \& Head and Neck Surgery, 7, 282-289. doi:10.1097/00020840-199910000-00012

Dubno, J. R., Dirks, D. D., \& Morgan, D. E. (1984). Effects of age and mild hearing loss on speech recognition in noise. Journal of the Acoustical Society of America, 76, 87-96. doi:10.1121/1.391011

Fitzgibbons, P. J., \& Gordon-Salant, S. (1996). Auditory temporal processing in elderly listeners. Journal of the American Academy of Audiology, 7, 183-189.

Fox, R. A. (1984). Effect of lexical status on phonetic categorization. Journal of Experimental Psychology: Human Perception and Performance, 10, 526-540. doi:10.1037/0096-1523.10.4.526

Ganong, W. F. (1980). Phonetic categorization in auditory word perception. Journal of Experimental Psychology: Human Perception and Performance, 6, 110-125. doi:10.1037/0096-1523.6.1.110

Gao, X., Levinthal, B. R., \& Stine-Morrow, E. A. L. (2012). The effect of ageing and visual noise on conceptual integration during sentence reading. The Quarterly Journal of Experimental Psychology, 65, 1833-1847. doi:10.1080/17470218.2012.674146

Gordon-Salant, S. (1987). Effects of acoustic modification on consonant recognition by elderly hearing-impaired subjects. Journal of the Acoustical Society of America, 81, 1199-1202. doi:10.1121/1.394643

Green, D. M., \& Swets, J. A. (1966). Signal detection theory and psychophysics. New York, NY: Wiley.

Guerreiro, M. J. S., Murphy, D. R., \& Van Gerven, P. W. M. (2010). The role of sensory modality in age-related inhibitory decline: A critical review. Psychological Bulletin, 136, 975-1022. doi:10.1037/a0020731

Hamm, V. P., \& Hasher, L. (1992). Age and the availability of inferences. Psychology and Aging, 7, 56-64. doi:10.1037/0882-7974.7.1.56

Hartley, A. A. (2001). Age differences in dual-task interference are localized to response-generation processes. Psychology and Aging, 16, 4754. doi:10.1037/0882-7974.16.1.47

Hartley, A. A., \& Little, D. M. (1999). Age-related differences and similarities in dual task interference. Journal of Experimental Psychology: General, 128, 416-449. doi:10.1037/0096-3445.128.4.416

Hartman, M., \& Hasher, L. (1991). Aging and suppression: Memory for previously relevant information. Psychology and Aging, 6, 587-594. doi:10.1037/0882-7974.6.4.587

Hasher, L., Stoltzfus, E. R., Zacks, R. T., \& Rypma, B. (1991). Age and inhibition. Journal of Experimental Psychology: Learning, Memory, and Cognition, 17, 163-169. doi:10.1037/0278-7393.17.1.163

Hasher, L., \& Zacks, R. T. (1988). Working memory, comprehension, and aging: A review and a new view. The Psychology of Learning and Motivation, 22, 193-225. doi:10.1016/S0079-7421(08)60041-9

Helfer, K. S., \& Wilber, L. A. (1990). Hearing loss, aging, and speech perception in reverberation and noise. Journal of Speech \& Hearing Research, 33, 149-155. 
Humes, L. E. (1996). Speech understanding in the elderly. Journal of the American Academy of Audiology, 7, 161-167.

Humes, L. E., \& Christopherson, L. (1991). Speech identification difficulties of hearing-impaired elderly persons: The contributions of auditory processing deficits. Journal of Speech \& Hearing Research, 34, 686693.

Humes, L. E., \& Dubno, J. R. (2010). Factors affecting speech understanding in older adults. In S. Gordon-Salant, R. D. Frisina, A. N. Popper, \& R. R. Fay (Eds.), The aging auditory system (pp. 211-257). New York, NY: Springer-Verlag. doi:10.1007/978-1-4419-0993-0_8

Humes, L. E., Lee, J. H., \& Coughlin, M. P. (2006). Auditory measures of selective and divided attention in young and older adults using singletalker competition. Journal of the Acoustical Society of America, 120, 2926-2937. doi:10.1121/1.2354070

Humes, L. E., Nelson, K. J., \& Pisoni, D. B. (1991). Recognition of synthetic speech by hearing-impaired elderly listeners. Journal of Speech \& Hearing Research, 34, 1180-1184.

Kawahara, H., Masuda-Katsuse, I., \& de Cheveigné, A. (1999). Restructuring speech representations using a pitch-adaptive time-frequency smoothing and an instantaneous-frequency-based F0 extraction: Possible role of a repetitive structure in sounds. Speech Communication, 27, 187-207. doi:10.1016/S0167-6393(98)00085-5

Kemper, S., \& Sumner, A. (2001). The structure of verbal abilities in young and older adults. Psychology and Aging, 16, 312-322. doi: 10.1037/0882-7974.16.2.312

Liberman, A. M., Harris, K. S., Hoffman, H. S., \& Griffith, B. C. (1957). The discrimination of speech sounds within and across phoneme boundaries. Journal of Experimental Psychology, 54, 358-368. doi:10.1037/ h0044417

MacKay, D. G., \& Burke, D. M. (1990). Cognition and aging: New learning and the use of old connections. In T. M. Hess (Ed.), Aging and cognition: Knowledge organization and utilization (pp. 213-263). Amsterdam, The Netherlands: North-Holland. doi:10.1016/S01664115(08)60159-4

Mattys, S. L., \& Wiget, L. (2011). Effects of cognitive load on speech recognition. Journal of Memory and Language, 65, 145-160. doi: 10.1016/j.jml.2011.04.004

McClelland, J. L., \& Elman, J. L. (1986). The TRACE model of speech perception. Cognitive Psychology, 18, 1-86.

McCoy, S. L., Tun, P. A., Cox, L. C., Colangelo, M., Stewart, R. A., \& Wingfield, A. (2005). Hearing loss and perceptual effort: Downstream effects on older adults' memory for speech. The Quarterly Journal of Experimental Psychology Section A: Human Experimental Psychology, 58, 22-33. doi:10.1080/02724980443000151

McDowd, J. M., \& Craik, F. I. M. (1988). Effects of aging and task difficulty on divided attention performance. Journal of Experimental Psychology: Human Perception and Performance, 14, 267-280. doi: 10.1037/0096-1523.14.2.267

McDowd, J. M., \& Shaw, R. J. (2000). Attention and aging: A functional perspective. In F. I. M. Craik and T. A. Salthouse (Eds.), The handbook of aging and cognition (2nd ed., pp. 221-292). Mahwah, NJ: Erlbaum Assoc.

Murphy, D. R., Daneman, M., \& Schneider, B. A. (2006). Why do older adults have difficulty following conversations? Psychology and Aging, 21, 49-61. doi:10.1037/0882-7974.21.1.49

Navon, D. (1984). Resources-A theoretical soup stone? Psychological Review, 91, 216-234. doi:10.1037/0033-295X.91.2.216

Navon, D., \& Gopher, D. (1979). On the economy of the human processing system. Psychological Review, 86, 214-255. doi:10.1037/0033-295X.86 .3 .214

Norris, D., McQueen, J. M., \& Cutler, A. (2000). Merging information in speech recognition: Feedback is never necessary. Behavioral and Brain Sciences, 23, 299-325. doi:10.1017/S0140525X00003241
Ohde, R. N., \& Abou-Khalil, R. (2001). Age differences for stop-consonant and vowel perception in adults. Journal of the Acoustical Society of America, 110, 2156-2166. doi:10.1121/1.1399047

Pichora-Fuller, M. K., Schneider, B. A., \& Daneman, M. (1995). How young and old adults listen to and remember speech in noise. Journal of the Acoustical Society of America, 97, 593-608. doi:10.1121/1.412282

Pichora-Fuller, M. K., \& Singh, G. (2006). Effects of age on auditory and cognitive processing: Implications for hearing aids fitting and audiologic rehabilitation. Trends in Amplification, 10, 29-59. doi:10.1177/ 108471380601000103

Pichora-Fuller, M. K., \& Souza, P. E. (2003). Effects of aging on auditory processing of speech. Journal of Audiology, 42(Suppl 2), 2S11--2S16.

Repp, B. H. (1984). Categorical perception: Methods, issues, findings. In J. Lass (Ed.), Speech and language: Advances in basic research and practice (Vol. 10, pp. 243-355). New York, NY: Academic Press.

Rönnberg, J., Danielsson, H., Rudner, M., Arlinger, S., Sternäng, O., Wahlin, A., \& Nilsson, L.-G. (2011). Hearing loss is negatively related to episodic and semantic long-term memory but not to short-term memory. Journal of Speech, Language, and Hearing Research, 54, 705-726. doi:10.1044/1092-4388(2010/09-0088)

Salthouse, T. A. (1996). The processing-speed theory of adult age differences in cognition. Psychological Review, 103, 403-428. doi:10.1037/ 0033-295X.103.3.403

Schneider, B. A., Daneman, M., \& Murphy, D. R. (2005). Speech comprehension difficulties in older adults: Cognitive slowing or age-related changes in hearing? Psychology and Aging, 20, 261-271. doi:10.1037/ 0882-7974.20.2.261

Schneider, B. A., Daneman, M., Murphy, D. R., \& Kwong-See, S. (2000). Listening to discourse in distracting settings: The effects of aging. Psychology and Aging, 15, 110-125. doi:10.1037/0882-7974.15.1.110

Schneider, B. A., Pichora-Fuller, M. K., \& Daneman, M. (2010). The effects of senescent changes in audition and cognition on spoken language comprehension. In S. Gordon-Salant, R. D. Frisina, A. Popper, \& D. Fay (Eds.), The aging auditory system: Springer handbook of auditory research (Vol. 34, pp. 167-210). Berlin, Germany: Springer. doi 10.1007/978-1-4419-0993-0_7

Schneider, B. A., Pichora-Fuller, M. K., Kowalchuk, D., \& Lamb, M (1994). Gap detection and the precedence effect in young and old adults Journal of the Acoustical Society of America, 95, 980-991. doi:10.1121/ 1.408403

Sommers, M. S. (1996). The structural organization of the mental lexicon and its contribution to age-related changes in spoken word recognition. Psychology and Aging, 11, 333-341. doi:10.1037/0882-7974.11.2.333

Sommers, M. S., \& Danielson, S. M. (1999). Inhibitory processes and spoken word recognition in young and older adults: The interaction of lexical competition and semantic context. Psychology and Aging, 14, 458-472. doi:10.1037/0882-7974.14.3.458

Souza, P. E., \& Turner, C. W. (1994). Masking of speech in young and elderly listeners with hearing loss. Journal of Speech \& Hearing Research, 37, 655-661.

Spieler, D. H., \& Balota, D. A. (2000). Factors influencing word naming in younger and older adults. Psychology and Aging, 15, 225-231. doi 10.1037/0882-7974.15.2.225

Strouse, A., Ashmead, D. H., Ohde, R. N., \& Grantham, D. W. (1998). Temporal processing in the aging auditory system. Journal of the Acoustical Society of America, 104, 2385-2399. doi:10.1121/1.423748

Suzman, R. M., Willis, D. P., \& Manton, K. G. (1992). The oldest old. New York, NY: Oxford University Press.

Takahashi, G. A., \& Bacon, S. P. (1992). Modulation detection, modulation masking, and speech understanding in noise in the elderly. Journal of Speech and Hearing Research, 35, 1410-1421.

Tipper, S. P. (1991). Less attentional selectivity as a result of declining inhibition in older adults. Bulletin of the Psychonomic Society, 29, 45-47. doi:10.3758/BF03334765 
Tun, P. A., O'Kane, G., \& Wingfield, A. (2002). Distraction by competing speech in younger and older listeners. Psychology and Aging, 17, 453467. doi:10.1037/0882-7974.17.3.453

Verhaeghen, P., Steitz, D. W., Sliwinski, M. J., \& Cerella, J. (2003). Aging and dual-task performance: A meta-analysis. Psychology and Aging, 18, 443-460. doi:10.1037/0882-7974.18.3.443

von Gunten, A., Ebbing, K., Imhof, A., Giannakopoulos, P., \& Kövari, E. (2010). Brain aging in the oldest-old. Current Gerontology and Geriatrics Research, 2010, article 358531. doi:10.1155/2010/358531

Wingfield, A., \& Tun, P. (2001). Spoken language comprehension in older adults: Interactions between sensory and cognitive change in normal aging. Seminars in Hearing, 22, 287-302. doi:10.1055/s-2001-15632

Wingfield, A., Tun, P. A., \& McCoy, S. L. (2005). Hearing loss in older adulthood: What it is and how it interacts with cognitive performance.
Current Directions in Psychological Science, 14, 144-148. doi:10.1111/ j.0963-7214.2005.00356.x

Wright, R. E. (1981). Aging, divided attention, and processing capacity. Journal of Gerontology, 36, 605-614. doi:10.1093/geronj/36.5.605

Yonan, C. A., \& Sommers, M. S. (2000). The effects of talker familiarity on spoken word identification in younger and older listeners. Psychology and Aging, 15, 88-99. doi:10.1037/0882-7974.15.1.88

Received June 14, 2012 Revision received July 24, 2013 Accepted August 12, 2013

\section{Correction to Lang et al. (2013)}

In the article "Forecasting life satisfaction across adulthood: Benefits of seeing a dark future?" by Frieder R. Lang, David Weiss, Denis Gerstorf, and Gert G. Wagner (Psychology and Aging, Vol. 28, No. 1, 249-261, doi: 10.1037/a0030797), there was an error in Equation 1 in the Methods section under the heading "Accuracy of anticipated future life satisfaction." The correct equation is as follows:

$$
\Delta \text { Accuracy }=F L S_{i}-C L S_{i+5}
$$

Please note that the correct equation was used in the statistical analyses as reported in the article.

DOI: $10.1037 / \mathrm{a} 0035890$ 